\title{
A Selective Algorithm for Wireless Temperature Monitoring of a Rotational Mold Casting Process with Surface Acoustic Wave (SAW) Sensors
}

\author{
Mykhaylo Yudytskiy ${ }^{1}$, Stefan Fischer ${ }^{1}$, René Fachberger ${ }^{1}$ \\ ${ }^{1}$ sensideon $\mathrm{GmbH}$, Carl-Blum-Str. 3, 4600 Wels, Austria \\ mykhaylo.yudytskiy@sensideon.com
}

\begin{abstract}
:
Wireless SAW sensor temperature measurement relies heavily on the quality of the transmitted signal. The standard approach of averaging consecutive signals to improve the quality is suboptimal in a rotational setting, where the sensor is temporarily invisible to the reader's antenna. In this paper we introduce a novel approach to pre-filtering the data by averaging a selection of useful signals. Three selection strategies are presented. By means of experiments we show that the method is superior to standard averaging. The algorithm particularly stands out when applied in an industrial application of rotational mold casting, where only ca. $10 \%$ of the data are useful. While the sensor is invisible to standard averaging, our algorithm makes process monitoring of up to around $300{ }^{\circ} \mathrm{C}$ possible.
\end{abstract}

Key words: surface acoustic waves, temperature sensors, signal processing algorithms.

\section{Introduction}

In a particular rotational mold casting process liquid metal is poured into a mold and spun at 1000-1500 rpm. To achieve optimal results, the mold must maintain a surface temperature of roughly $300{ }^{\circ} \mathrm{C}$. Fire and water are applied to the surface of the mold to regulate its temperature.

High temperatures and severe environmental conditions (dirt, vibrations, and contact with fire and water) reduce the scope of applicable technologies to a handful. Surface acoustic wave (SAW) sensors are a particularly good choice: they are a very robust, precise, and passive instrument for measuring temperature wirelessly [1],[2]. In addition they are practically maintenance and calibration-free. A SAW sensor is queried by a reader unit. Response signals are evaluated to obtain a sensor value.

In order to unleash the full potential of SAW technology in the industrial setting described above, several challenges must be overcome. First, wirelessly transmitted data is inherently contaminated with noise, the presence of which deteriorates the accuracy of the SAW temperature measurement. Additional problem arises due to rotation, since the sensor is not permanently visible to the reader unit. Beside the signal-related impediments, the sensor must be designed to withstand contact with fire, water and hot steam.
Signal processing-based concepts can significantly improve the measurement quality of SAW sensors [3],[4],[5]. The focus of this work lies on a novel algorithm to filter consecutive response signals. The procedure consists of two essential steps. First, from a set of consequent measurements the algorithm selects only those measurements that contain valuable information. Next, average of the selection is used to compute the sensor value. Several ideas on selection criteria were investigated in this work.

This technique is especially useful in applications where the sensor is temporarily invisible to the reader antenna, e.g., during rotation. Signal quality can be improved tremendously over simply averaging the data. This approach is also superior to a triggerbased system, as no external device is needed.

The paper is structured in the following way. First we describe the standard averaging approach and set the notation. Next, we present three alternative selective averaging techniques with the goal of improving signal quality in a rotational setting. Then, we demonstrate the performance of the three methods and compare them with the standard averaging technique by means of two examples: a laboratory experiment and the industrial application described above. Finally, we state our conclusions and outlook. 


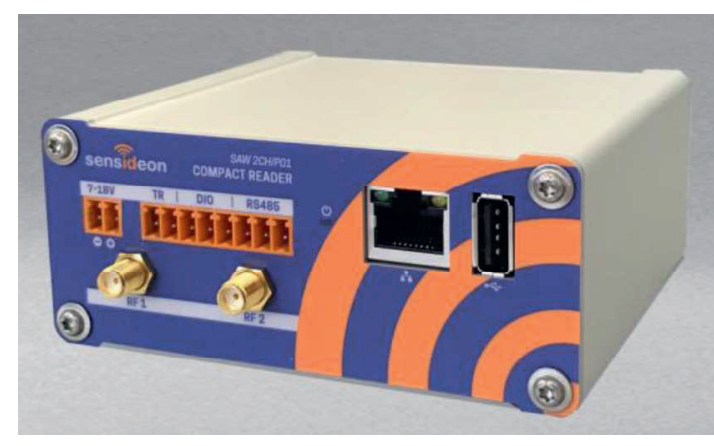

Fig.1. sensideon reader unit.

\section{Problem Formulation}

The interaction between the reader unit (see Fig. 1) and a delay-line SAW tag (see Fig. 2) may be described in the following way. The interrogation unit queries the tag by sending a sequence of $N$ impulse signals at frequencies $f_{n}=f_{0}+n B / N$, for $n=0, \ldots, N-1$, where $f_{0}$ is the starting frequency and $B$ is the bandwidth. At each frequency step $n$, a response signal is received by the reader.

The delay-line SAW tag is outfitted with $r$ reflectors. The response signal is a superposition of $r$ reflections at different delay times $\tau_{1}, \ldots, \tau_{r}$. The model of the response signal of a tag with $r$ reflectors at frequency step $n$ is given by

$$
S_{*}[n]=\sum_{j=1}^{r} A_{j} \exp \left(2 \pi i f_{n} \tau_{j}\right),
$$

where $\tau_{j}$ and $A_{j}$ are the round trip delay time (RTDT) and the amplitude of reflection $j=1, \ldots, r$, respectively. More details can be found in, e.g., [6],[7],[8]. We write the response in vector form $S_{*}=\left(s_{*}[0], \ldots, S_{*}[N-1]\right)^{T}$.

Any wireless measurement is contaminated by noise. We assume additive noise and write $s=s_{*}+\eta$, where the measured signal $s$ is a combination of the true signal $S_{*}$ and a noise component $\eta$, which is a complex vector of dimension $N$. In this paper we will not go into the nature of the noise, part of which is random white noise and part of which has a structure.

Several algorithms exist to extract radio frequency identification (RFID) [8],[9] and sensor quantities [3],[4],[5],[7],[10] from $s$, even in presence of large measurement noise. A typical way to reduce the noise level is to average several consecutive measurements. This type of averaging is typically part of the reader firmware.

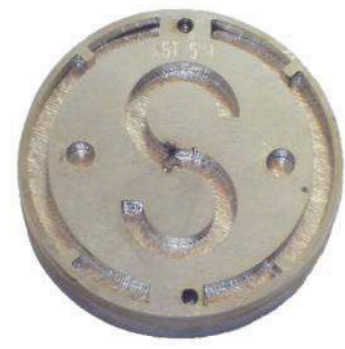

Fig.2. sensideon sensor. Dimensions: $50 \mathrm{~mm}$ diameter, $11 \mathrm{~mm}$ height. An M10 screw on the back allows for easy installation.

Let us denote $m$ consecutive measurements by $s_{1}, \ldots, s_{m}$. To improve the signal quality, the data is averaged,

$\bar{s}=\frac{1}{m} \sum_{i=1}^{m} s_{i}$.

In a typical application, this will increase the signal to noise ratio (SNR).

In this paper we focus on a different application: the tag is attached to a rotating object and is partially invisible to the reader's antenna during rotation. In this case, not all consecutive measurements $s_{i}$ will contain useful signal information. Averaging all measurements in this setting is counterproductive. The effect worsens at lower rotational speeds, as fewer measurements contain useful information.

Ideally, in this situation we want to select a subset of good measurements and discard the measurements that have no or little useful information. The problem can be formulated as follows: find a subset of measurements indexed by $I \subseteq\{1, \ldots, m\}$, where all measurements $s_{i}$ with $i \in I$ contain useful signal information.

The average of the selection is given by $\hat{s}=\frac{1}{|I|} \sum_{i \in I} s_{i}$

where $|I|$ denotes the number of elements of set $I$. What constitutes a useful measurement and how to select the set $I$ in practice is discussed in the following sections.

\section{Selective Algorithms}

We will present three alternative strategies for selecting a subset of good measurements from a consecutive set of $m$ signals below. Before going into the details of each method, let us introduce the notation. 
Consider two complex-valued vectors of dimension $\quad N, \quad a=(a[0], a[1] \ldots, a[N-1])^{T}$ and $b=(b[0], b[1], \ldots, b[N-1])^{T}$. We write. to denote the component-wise multiplication,

$a \cdot b=(a[0] b[0], \ldots, a[N-1] b[N-1])^{T}$.

The scalar product is given by

$$
\langle a, b\rangle=a[0] \overline{b[0]}+\ldots+a[N-1] \overline{b[N-1]},
$$

where the bar denotes the complex conjugate. The norm of a vector is denoted by $\|a\|=\sqrt{\langle a, a\rangle}$.

The RTDTs necessary for computing the RFID or sensor values, appear as distinct peaks in the time domain of the signal. Typically (cf. [3]), a zero-padded windowed Fourier transform is used to transform the signal from the frequency domain to the time domain. Let $w$ (vector of dimension $N$ ) denote the windowing function (e.g., the Hamming function) and $F$ be the (zero-padded) Fourier transform. The signal in time domain is given by

$v=F(w \cdot s)$.

We write $v_{i}=F\left(w \cdot s_{i}\right)$ for $i=1, \ldots, m$ to denote the measured signal in time domain. Note that in several algorithm steps below, by applying the Plancherel theorem, the Fourier transform does not need to be computed explicitly. This improves the computational complexity of the methods.

\section{Selection by Comparing with Average Signal}

The first approach that we introduce is based on comparing the individual measurements with the average signal. This approach is applicable if the average signal contains sufficient information on the reflector positions, but is, e.g., too weak for an exact temperature evaluation.

The average signal in time domain is given by

$\bar{v}=\frac{1}{m} \sum_{i=1}^{m} v_{i}$.

We use the normalized scalar product of two signals to quantify the similarity between them. We say two signals are similar if their normalized scalar product is close to the value one.

Let $\alpha \in[0,1]$ be a fixed parameter. The signals similar to the average are indexed by
$I=\left\{i \in\{1, \ldots, m\}: \alpha \leq \frac{\left\langle\bar{v}, v_{i}\right\rangle}{\|\bar{v}\|\left\|v_{i}\right\|}\right\}$.

Parameter $\alpha$ should be chosen such that the scalar product with signals containing mostly noise is below the threshold.

\section{Selection by Signal Strength and Similarity}

The second approach is based on selecting data by signal strength and similarity. The method consists of two steps. First, we select the signals with the largest magnitude. This is motivated by the assumption that the signal component is prevalent to noise. Second, we group those signals according to similarity and choose the largest group. As in the previous method, we use the normalized scalar product to compare similarity of signals.

Let $\alpha, \beta \in[0,1]$ be two fixed parameters. The set of signals with largest magnitude is given by

$$
I_{0}=\left\{i \in\{1, \ldots, m\}: \alpha \leq \frac{\left\|v_{i}\right\|}{\max _{j=1, \ldots, m}\left\|v_{j}\right\|}\right\},
$$

i.e., the norm of the signal is at least as large as the fraction $\alpha$ of the largest norm. In the set $I_{0}$, the number of signals similar to $v_{i}$ is given by

$$
f(i)=\left|\left\{j \in I_{0}: \beta \leq \frac{\left\langle v_{i}, v_{j}\right\rangle}{\left\|v_{i}\right\|\left\|v_{j}\right\|}\right\}\right|,
$$

for $i \in I_{0}$. Parameter $\beta$ is the similarity threshold. The signal with most similarities is the (possibly non-unique) maximum of $f$,

$$
k=\underset{i \in I_{0}}{\arg \max } f(i) \text {. }
$$

Finally, all of the signals in the group with the most similar signals are indexed by

$$
I=\left\{j \in I_{0}: \beta \leq \frac{\left\langle v_{k}, v_{j}\right\rangle}{\left\|v_{k}\right\|\left\|v_{j}\right\|}\right\} .
$$

As will be shown in the results section, the assumption that the magnitude of the signal is larger than noise does not always hold in practice. Strong interference will cause the algorithm to lock onto a bad set of measurements.

\section{Selection by Evaluating Individual Signals}

Finally, the last approach that we introduce is based on selecting similar plausible measurements. We can decide if an individual measurement signal is plausible if certain 
conditions, such as the number of detected peaks, signal to noise ratio, an RFID checksum, etc., are fulfilled.

In this approach, we first compute the average of the plausible measurements. Individual measurements are then compared to this average and signals similar to the average are selected for the final set.

We use a different criterion for comparing the signals with the average. Instead of scalar products, as in the methods above, we compare RTDTs and peak amplitudes, as these are computed for the plausibility checks. In contrast to the previous two methods, this approach is more computationally demanding.

Let $I_{0} \subseteq\{1, \ldots, m\}$ be the subset of indices of plausible measurements. We construct an average using the plausible measurements,

$$
v_{0}=\frac{1}{\left|I_{0}\right|} \sum_{i \in I_{0}} v_{i} \text {. }
$$

Next, we compute the approximate RTDTs and the peak amplitudes of the average $v_{0}$ as well as of the individual signals $v_{i}$ (using, e.g., the method described in [3]). We denote the RTDT and the amplitude of reflector $j=1, \ldots, r$ and index $i=0,1, \ldots, m$ by $\tau_{j}^{(i)}$ and $A_{j}^{(i)}$, respectively.

Finally, let $\alpha, \beta \geq 0$ be two fixed constants. We select a subset of the plausible measurements,

$$
\begin{aligned}
I=\left\{i \in I_{0}:\right. & \min _{j=1, \ldots, r} A_{j}^{(i)} / A_{j}^{(0)}>\alpha \text { and } \\
& \left.\max _{j=1, \ldots, r}\left|\tau_{j}^{(i)}-\tau_{j}^{(0)}\right|<\beta / 2\right\},
\end{aligned}
$$

i.e., signals where all peaks are larger than factor $\alpha$ of the average and all RTDTs are in the $\beta$-vicinity of the average RTDTs. This way we make sure that all signals in the set are similar to the average of plausible measurements and have strong peaks.

\section{Measurement Setup}

We tested the quality of each method by two means: in a laboratory experiment and in a rotational mold casting process, described in the beginning of this work. Here we give a brief overview of the measurement setup. In the next sections we state the results.

The measurements were performed using a sensideon four channel reader (the two channel version is depicted in Fig. 1). The tag was interrogated with a starting frequency of $f_{0}=2.4 \mathrm{GHz}$ over the bandwidth of roughly $B=80 \mathrm{MHz}$. Two sweeps were used per measurement. The measurement time was approximately $10 \mathrm{~ms}$. A total of $m=80$ consecutive measurements were used.

Due to the extremely severe demands of the measurement environment in the mold casting process, a special sensor had to be used, see Fig. 2. The sensor was designed to be fireproof and waterproof. This way, the sensor could withstand direct contact with flame during the heating process and the SAW tag was not subject to corrosive effects of hot steam during the cooling process.

The mold with an integrated sensor is shown in Fig. 3. Liquid metal is poured into the mold from the side. The mold is spun at $1000-1500 \mathrm{rpm}$. The surface of the mold and the sensor are subject to flames during the heating process and to water during the cooling process.

In the laboratory setting, we attached a tag to a rotating object. Part of the path of the tag was shielded from the reader's antenna, simulating the temporary invisibility of the industrial setting. Rotational speed was set to match the 1000 $1500 \mathrm{rpm}$ of the mold casting process. The measurement was performed at room temperature.

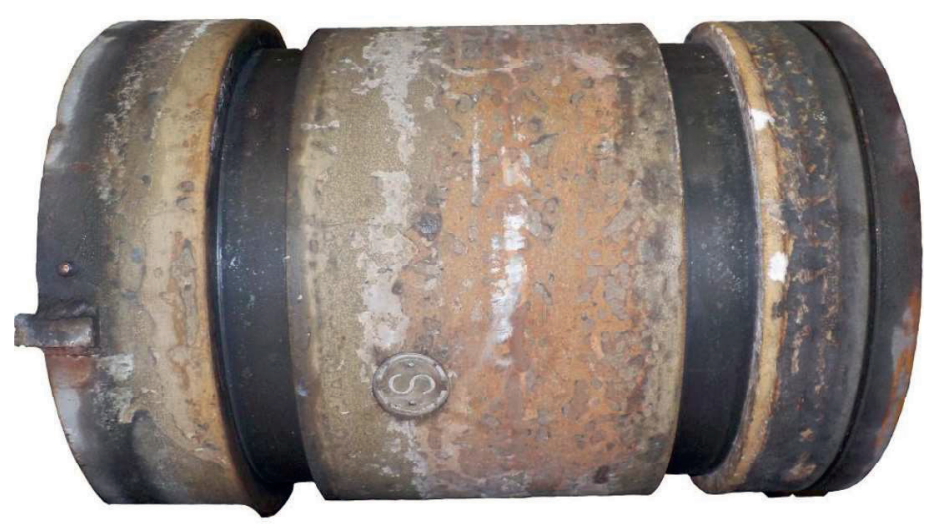

Fig.3. Mold for rotational casting with an integrated sensideon sensor (lower middle section of the image). 
The three algorithms were configured in the same way for both tests. See Table 1 for more details.

Tab. 1: Algorithm configurations. Parameter $\beta$ of the selection by evaluating individual signals algorithm, marked with star $\left({ }^{*}\right)$, was set to the default grid spacing of the tag's reflectors.

\begin{tabular}{|c|c|c|}
\hline Algorithm & $\alpha$ & $\beta$ \\
\hline Comparing with average signal & 0.7 & \\
\hline Signal strength and similarity & 0.9 & 0.9 \\
\hline Evaluating individual signals & 0.95 & $*$ \\
\hline
\end{tabular}

\section{Measurement Results: Laboratory}

In the laboratory experiment, the measurement data was collected over a period of a few minutes. Typical signal data observed during the test (in time domain, $v_{i}$ ) is plotted in Fig. 4.

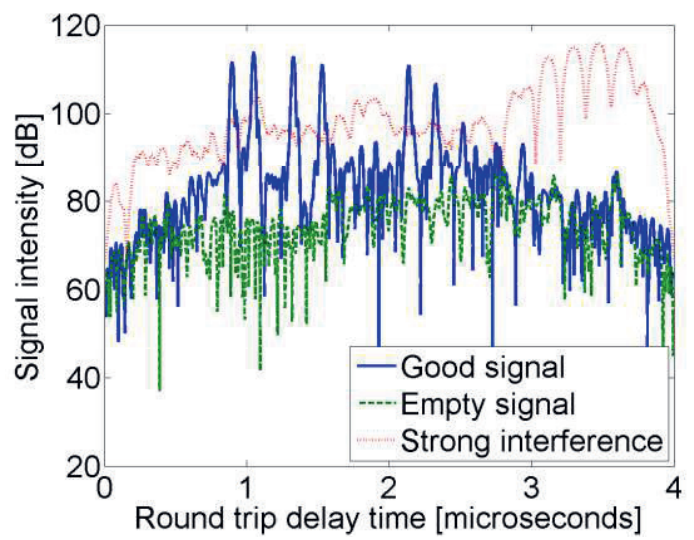

Fig.4. Three types of typical measurement signals: good signal (solid blue line), empty signal, i.e., pure noise (dashed green line), and strong external interference (dotted red line).

During rotation the signal was either visible (solid blue line) or invisible (dashed green line) to the reader's antenna. Occasional strong external interference was observed (dotted red line).

Comparison between standard and selective averaging is plotted in Fig. 5; selection by evaluating individual signals was used. As can be seen from the plot, the signal with selective averaging is stronger than that of standard averaging. Roughly half of the 80 measurements were selected.

Quantitatively, performance can be compared using the SNR. Average SNR of the different methods are given in Table 2. Higher SNR means better signal quality.

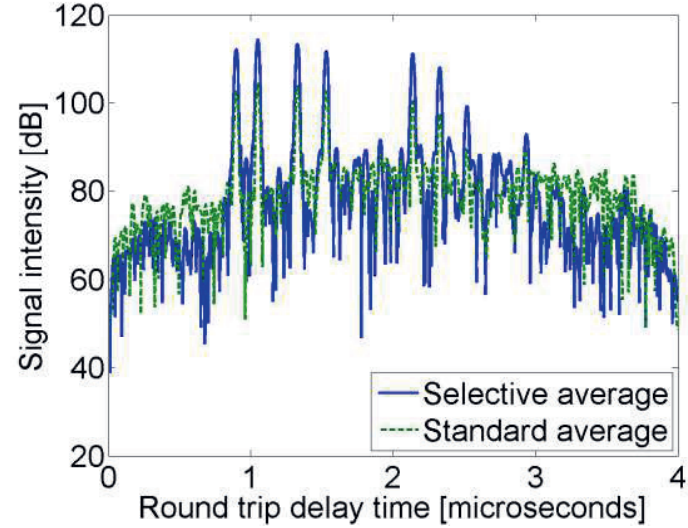

Fig.5. Performance comparison between selective averaging (solid blue line) and standard averaging (dashed green line) in the lab experiment.

Tab. 2: Signal to noise ratio (SNR) using the data from the lab.

\begin{tabular}{|c|c|}
\hline Algorithm & SNR [dB] \\
\hline Standard averaging & 22.41 \\
\hline Comparing with average signal & 36.84 \\
\hline Signal strength and similarity & N/A \\
\hline Evaluating individual signals & 39.19 \\
\hline
\end{tabular}

The best results were obtained with the third algorithm (evaluating individual signals). Significant improvement of selective averaging over standard averaging was observed.

Selection by signal strength and similarity failed in this test due to the occasional strong external interference. The algorithm locked on interference signals, rather than on tag data, as they had the largest norm.

\section{Measurement Results: Mold Casting}

In the rotational mold casting process, data was collected for a duration of about an hour. A comparison between the standard and selective averaging is plotted in Fig. 6; selection by evaluating individual signals was used.

The typical number of selected signals was eight out of $80(10 \%)$. For standard averaging, the amount of data was insufficient, as can be seen in Fig. 6. In comparison, the quality of the signal produced by selective averaging was adequate for temperature evaluation.

As a result, neither standard averaging, nor selection by comparing with average signal were successful, due to the poor quality of the average signal. Results from the other two selective averaging algorithms are plotted in Fig. 7. Due to confidentiality, temperature is given in arbitrary units. 


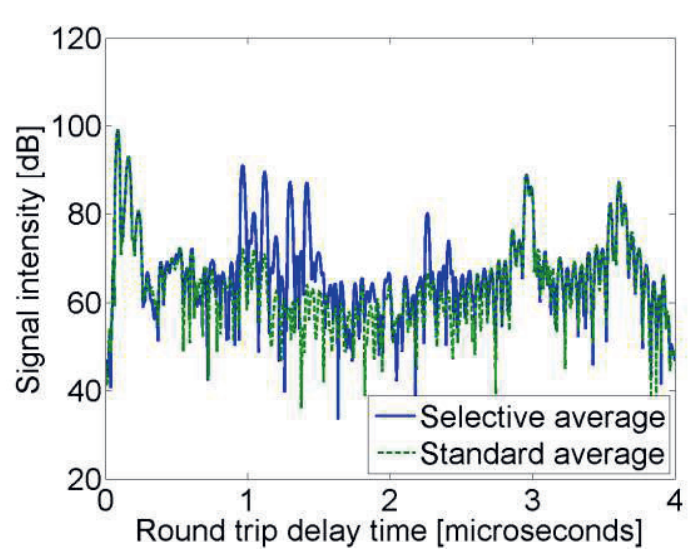

Fig.6. Performance comparison between selective averaging (solid blue line) and standard averaging

(dashed green line) in the industrial setting.

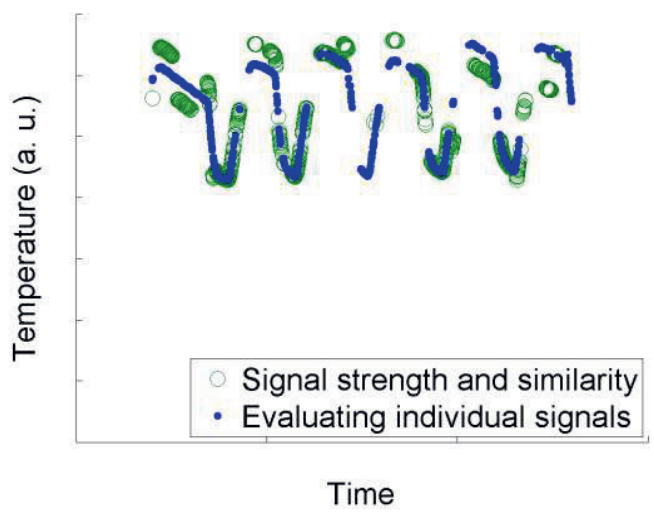

Fig.7. Temperature measurement of the rotational mold casting process. Results of selection by signal strength and similarity (hollow green circles) and by evaluating individual signals (solid blue circles) are shown. Temperature range is ca. 200 to $300^{\circ} \mathrm{C}$.

Results of the remaining two methods are as follows. At lower temperatures, both methods performed comparably well. At higher temperatures, data selected by signal strength and similarity were qualitatively not good, leading to erroneous temperature values.

Selection by evaluating individual signals was the only method to produce accurate temperature results. Although short intervals where no measurements were possible exist, the overall structure of the process, with distinct minima and maxima in the cycle, is clearly visible.

\section{Conclusion and Outlook}

In this work we presented three innovative methods to improve the signal quality of a wireless SAW sensor measurement in a rotational setting by selective averaging. The three methods were: (1) selection by comparing with average signals, (2) selection by signal strength and similarity and (3) selection by evaluating individual signals.
We examined the performance of each method by two means: in a laboratory experiment and in a rotational mold casting process. The benefit of using a selective algorithm instead of the standard averaging was clearly demonstrated. In fact, without selective averaging no measurements could be obtained for the mold casting process.

The third method (evaluating individual signals) performed best in both tests. The other two methods showed drawbacks in one or both tests. They can be improved by supplementing them with additional checks in future work.

\section{References}

[1] A. Pohl, A review of wireless SAW sensors, IEEE Tran. on Ultrasonics, Ferrorel., and Freq. Control 47, 317 (2000); doi: 10.1109/58.827416

[2] L. Reindl, A. Pohl, G. Scholl, R. Weigel, SAWbased radio sensor systems, IEEE Sensors Journal 1 (2001); doi:10.1109/JSEN.2001.923589

[3] L. Reindl, I. Shrena, Wireless measurement of temperature using surface acoustic waves sensors, IEEE Tran. on Ultrasonics, Ferrorel., and Freq. Control 51, 11 (2004); doi: 10.1109/TUFFC.2004.1367486

[4] S. Schuster, S. Scheiblhofer, A. Stelzer, A Springer, Model based wireless SAW tag temperature measurement, APMC (2005); doi: 10.1109/APMC.2005.1606436

[5] M. Yudytskiy and R. Fachberger, Continuous temperature monitoring algorithm for SAW sensors, 2015 IEEE Intl. Ultrasonics Symposium (2015); doi: 10.1109/ULTSYM.2015.0460

[6] S. Scheiblhofer, S. Schuster, A. Stelzer, Signal model and linearization for nonlinear chirps in FMCW radar SAW-ID tag request, IEEE Tran. on Microwave Theory and Techniques 54, 1477 (2006); doi: 10.1109/TMTT.2006.871361

[7] S. Schuster, S. Scheiblhofer, L. Reindl, A. Stelzer, Performance evaluation of algorithms for SAW-based temperature measurement, IEEE Tran. on Ultrasonics, Ferrorel., and Freq. Control 53 (2006); doi:10.1109/TUFFC.2006.1642516

[8] M. Klaffenböck, S. Schuster, S. Scheiblhofer, A. Stelzer, Model-based identification method for pulse position coded surface acoustic wave identification tags, IEEE Tran. on Instr. and Meas. 63, 2405 (2014); doi: 10.1109/TIM.2014.2310032

[9] V. Plessky and L. Reindl, Review on SAW RFID tags, IEEE Tran. on Ultrasonics, Ferrorel., and Freq. Control 57, 654 (2010); doi: 10.1109/TUFFC.2010.1462

[10] R. Fachberger, G. Bruckner, R. Hauser, and L. Reindl, Wireless SAW based high-temperature measurement systems, 2006 IEEE International Freq. Control Symposium and Exposition (2006); doi: 10.1109/FREQ.2006.275412 